\title{
Automatic Matching of Resource Needs and Availabilities in Microblogs for Post-Disaster Relief
}

\author{
Moumita Basu \\ UEM Kolkata, India; IIEST Shibpur, India \\ Kripabandhu Ghosh \\ IIT Kanpur, India
}

\begin{abstract}
During a disaster event, it is essential to know about needs and availabilities of different types of resources, for coordinating relief operations. Microblogging sites are frequently used for aiding post-disaster relief operations, and there have been prior attempts to identify tweets that inform about resource needs and availabilities (termed as need-tweets and availability-tweets respectively). However, there has not been much attempt to effectively utilise such tweets. We introduce the problem of automatically matching need-tweets with appropriate availability-tweets, which is practically important for coordination of post-disaster relief operations. We also experiment with several methodologies for automatically matching need-tweets and availability-tweets.
\end{abstract}

\section{CCS CONCEPTS}

- Information systems $\rightarrow$ Information retrieval;

\section{KEYWORDS}

Disaster; Microblogs; Resource needs and availabilities; Matching

ACM Reference Format:

Moumita Basu, Anurag Shandilya, Kripabandhu Ghosh, and Saptarshi Ghosh. 2018. Automatic Matching of Resource Needs and Availabilities in Microblogs for Post-Disaster Relief. In WWW'18 Companion: The 2018 Web Conference Companion, April 23-27, 2018, Lyon, France. ACM, New York, NY, USA, 2 pages. https://doi.org/10.1145/3184558.3186911

\section{INTRODUCTION}

In a disaster situation (e.g., earthquake, flood), two types of information that are especially useful for coordinating relief operations are what resources are needed and what resources are available in the affected region $[1,3]$. Microblogging sites like Twitter are very useful for gathering situational information during disasters [2]. Hence, it is a natural quest to obtain information about post-disaster needs and availabilities of resources from microblogs. Specifically, two types of tweets (microblogs) are useful: (i) Need-tweets, which inform about the need or requirement of specific resources, e.g., food, water, medical aid, shelter, and (ii) Availability-tweets, which inform about the availability of resources in the affected region, or potential availability i.e. resources being transported to the region.

This paper is published under the Creative Commons Attribution 4.0 International (CC BY 4.0) license. Authors reserve their rights to disseminate the work on their personal and corporate Web sites with the appropriate attribution.

WWW'18 Companion, April 23-27, 2018, Lyon, France

(C) 2018 IW3C2 (International World Wide Web Conference Committee), published under Creative Commons CC BY 4.0 License.

ACM ISBN 978-1-4503-5640-4/18/04.

https://doi.org/10.1145/3184558.3186911

\author{
Anurag Shandilya \\ IIT Kharagpur, India
}

Saptarshi Ghosh

IIT Kharagpur, India; IIEST Shibpur, India

\begin{tabular}{|l|l|}
\hline Need-tweet (excerpts) & Availability-tweet (excerpts) \\
\hline $\begin{array}{l}\text { Mobile phones are not work- } \\
\text { ing, no electricity, no water in } \\
\text { \#Thamel, \#Nepalquake }\end{array}$ & $\begin{array}{l}\text { Please contact for drinking free service water } \\
\text { specially for Earthquake Victim. Sanjay Limbu } \\
\text { [mobile num] }\end{array}$ \\
\hline $\begin{array}{l}\text { Over 1400 killed. Many } \\
\text { Trapped. Medical Supplies } \\
\text { Requested. }\end{array}$ & $\begin{array}{l}\text { 20,000 RSS personnel with medical supplies } \\
\text { and other help the first to reach earthquake } \\
\text { damaged zones in \#Nepal }\end{array}$ \\
\hline $\begin{array}{l}\text { Nepal earthquake: thousands } \\
\text { in need of shelter in country } \\
\text { little able to cope [url] }\end{array}$ & $\begin{array}{l}\text { can anyone we know pick the 2000 second hand } \\
\text { tents from Sunauli and distribute it to the peo- } \\
\text { ple in need in Nepal? }\end{array}$ \\
\hline
\end{tabular}

Table 1: Examples of need-tweets and matching availabilitytweets, from a dataset of tweets on 2015 Nepal earthquake. The common resource for each pair is shown in bold.

For helping the relief operations, the first challenge is to extract need-tweets and availability-tweets from among the thousands of tweets posted during a disaster situation, most of which contain conversational content. This problem of identifying need-tweets and availability-tweets was addressed in our prior works [1, 3]. However, to our knowledge, apart from identification, there has not been much attempt to effectively utilise such tweets for postdisaster relief operations. In this work, we take the first step towards this goal, by automatically matching resource needs with appropriate availabilities. We assume that a set of need-tweets and a set of availability-tweets have already been identified from a given (large) set of tweets, and we focus on the matching of the identified need-tweets with the availability-tweets.

The actual problem of matching resource needs and availabilities is a complex one, which should consider several factors like the specific resource which is needed / available, the time at which it is needed / available, the specific geographical location of the need / availability, and so on. In this work, we address a simplified version of the matching problem - we assume that an availabilitytweet matches a need-tweet if and only if they inform about the need and availability of the same resource. Note that even this simplified version of the matching problem can be challenging. For instance, Table 1 shows some examples of need-tweets and matching availability-tweets, from one of the datasets used in this work. While some pairs might be easier to match since they state exactly the same resource (e.g., 'water' or 'medical supplies'), some pairs are much more difficult to match because they use different terms to refer to the same resource (e.g., need of 'shelter' and availability of 'tents', as shown in Table 1).

We experiment with several methodologies for matching needtweets and availability-tweets, ranging from simple noun overlap to methods using word embeddings. Though the methods achieve some success, it becomes evident that the problem of matching needs and availabilities needs to be explored further in future. 


\section{METHODOLOGIES FOR MATCHING NEEDS AND AVAILABILTIES}

For a particular need-tweet, we identify a list of matching availabilitytweets, ranked in decreasing order of how closely they match the need-tweet. We use several methodologies for such matching.

(1) Matching based on common nouns: We use a part-of-speech tagger on need-tweets and availability-tweets to identify the nouns, since nouns are likely to indicate the specific resources that are needed / available. To check the match of an availability-tweet with a need-tweet, we compute the fraction of the nouns in the needtweet that are also contained in the availability-tweet. For a given need-tweet, availability-tweets are ranked in the decreasing order of the fraction of common nouns (ties resolved arbitrarily).

(2) Matching based on local word embeddings: Here we train Word2vec [4] on the set of tweets from where the need-tweets and availability-tweets have been identified. We construct a tweetvector for each tweet, by averaging the Word2vec term-vectors of the terms contained in the tweet. The match between an availabilitytweet and a need-tweet is given by the cosine similarity of their tweet-vectors. For a given need-tweet, availability-tweets are ranked in the decreasing order of this cosine similarity value.

(3) Matching based on pre-trained word embeddings: A limitation of methodology (2) is that a large set of tweets about the current event is needed to learn the local word embeddings. At times of a disaster, it might be necessary to start matching needs and availabilities even before a sizeable number of tweets about the current event have been collected. To this end, we explore the use of three types of pre-trained embeddings.

(3a) Pre-trained GloVe embeddings [5], which are pre-trained on two billion tweets from the Twitter 1\% random sample [5].

(3b) Pre-trained embeddings for detecting paraphrases in tweets [6]: These embeddings are designed to detect paraphrases in tweets, i.e., tweets which are lexically different but semantically similar [6]. Hence, these embeddings are likely to identify needs and availabilities that might be written differently, but have similar semantics.

(3c) Word2vec embeddings pre-trained over tweets related to several disaster or crisis events [2], which can be expected to capture well the semantics of terms used during disaster events.

For all the pre-trained embeddings, we follow a matching methodology similar to (2), where we compute a tweet-vector for each tweet, and then compute the cosine similarity between the tweet-vectors of need-tweets and availability-tweets.

\section{EXPERIMENTS AND RESULTS}

Datasets: We used the Twitter API to collect English tweets related to two disaster events - (i) 50,018 tweets posted after the 2015 Nepal earthquake, and and (ii) 70,487 tweets posted after the 2016 earthquake in Italy. We refer to the datasets as nepal-quake and italy-quake respectively. We then used three human annotators to identify need-tweets and availability-tweets. For the nepal-quake dataset, they found 499 need-tweets and 1333 availability-tweets; for the italy-quake dataset, 177 need-tweets and 233 availabilitytweets were found. Details of the datasets can be found in our prior works [1, 3].

Evaluation measures: For a given need-tweet, each methodology produces a ranked list of availability-tweets, evaluated as follows.

\begin{tabular}{|l||c|c||c|c|}
\hline \multirow{2}{*}{ Methodology for matching } & \multicolumn{2}{|c||}{ Nepal-quake } & \multicolumn{2}{c|}{ Italy-quake } \\
\cline { 2 - 5 } & Prec & Recall & Prec & Recall \\
\hline Common noun overlap & 0.535 & 0.832 & $\mathbf{0 . 6 7 3}$ & $\mathbf{0 . 9 2 4}$ \\
\hline Local Word2vec embeddings & $\mathbf{0 . 7 6 8}$ & $\mathbf{0 . 9 5 4}$ & 0.400 & 0.773 \\
\hline Glove embeddings [5] & 0.190 & 0.568 & 0.285 & 0.697 \\
\hline Paraphrase embeddings [6] & 0.262 & 0.677 & 0.400 & 0.773 \\
\hline Crises-specific embeddings [2] & 0.276 & 0.715 & 0.303 & 0.712 \\
\hline
\end{tabular}

Table 2: Evaluation of matching need-tweets and availability-tweets over the two datasets. Best values are in boldface.

(i) Precision of matching: For each need-tweet, we used each methodology to retrieve 5 top-matching availability-tweets. We then asked the human annotators to check each need-availability pair, and judge whether the match is correct. There was very high agreement between all the annotators; majority voting was considered in the few cases where there was no unanimous agreement. The precision of a matching methodology is the fraction of pairs that are matched correctly by the methodology. In Information Retrieval terminology, we are computing Precision@5.

(ii) Recall of matching: The recall of a methodology is the fraction of all need-tweets for which the methodology is able to identify at least one correct matching (based on the judgement of the annotators). Evaluation results: Table 2 compares the performance of the various methodologies for the two datasets. We see that the methodology based on noun overlap performs reasonably well; in fact, this method performs the best for the italy-quake dataset. Pre-trained word embeddings, including embeddings trained on crises-related tweets, perform worse than the noun overlap methodology for both datasets, probably because a new event is associated with a very different vocabulary than prior events (on which the models are pre-trained). The methodology using local Word2vec embeddings (trained on the tweets of the current event) performs the best for nepal-quake dataset, but worse than the common noun overlap method for the italy-quake dataset. We will investigate the causes for this difference in performances in future.

\section{CONCLUSION}

We introduced the problem of matching post-disaster resource needs and availabilities from microblogs, which is important for effective coordination of post-disaster relief operations. We found that, even for a simplified version of the problem, more effective methods are necessary, especially for tackling the varied vocabulary of different disaster events. We plan to study more realistic versions of the matching problem in future.

\section{REFERENCES}

[1] Moumita Basu, Kripabandhu Ghosh, Somenath Das, Ratnadeep Dey, Somprakash Bandyopadhyay, and Saptarshi Ghosh. 2017. Identifying Post-Disaster Resource Needs and Availabilities from Microblogs. In Proc. ASONAM.

[2] Muhammad Imran, Prasenjit Mitra, and Carlos Castillo. 2016. Twitter as a Lifeline: Human-annotated Twitter Corpora for NLP of Crisis-related Messages. In Proc. LREC.

[3] Prannay Khosla, Moumita Basu, Kripabandhu Ghosh, and Saptarshi Ghosh. 2017. Microblog Retrieval for Post-Disaster Relief: Applying and Comparing Neural IR Models. CoRR abs/1707.06112 (2017). arXiv:1707.06112

[4] T. Mikolov, W.T. Yih, and G. Zweig. 2013. Linguistic Regularities in Continuous Space Word Representations. In NAACL HLT 2013.

[5] Jeffrey Pennington, Richard Socher, and Christopher D. Manning. 2014. GloVe: Global Vectors for Word Representation. In Proc. EMNLP.

[6] Wei Xu, Alan Ritter, Chris Callison-Burch, William B. Dolan, and Yangfeng Ji. 2014. Extracting Lexically Divergent Paraphrases from Twitter. TACL 2, 1 (2014). 